Documentation et bibliothèques

DOCUMENTATION BIBLIOTHËQUES

\title{
Pistes de solutions pour l'amélioration des relations entre les services techniques et les services au public
}

\section{Johanne Lavoie}

Volume 52, numéro 1, janvier-mars 2006

URI : https://id.erudit.org/iderudit/1030028ar

DOI : https://doi.org/10.7202/1030028ar

Aller au sommaire du numéro

Éditeur(s)

Association pour l'avancement des sciences et des techniques de la

documentation (ASTED)

ISSN

0315-2340 (imprimé)

2291-8949 (numérique)

Découvrir la revue

Citer cet article

Lavoie, J. (2006). Pistes de solutions pour l'amélioration des relations entre les services techniques et les services au public. Documentation et bibliothèques,

52(1), 59-65. https://doi.org/10.7202/1030028ar

Tous droits réservés (c) Association pour l'avancement des sciences et des techniques de la documentation (ASTED), 2006
Ce document est protégé par la loi sur le droit d'auteur. L'utilisation des services d’Érudit (y compris la reproduction) est assujettie à sa politique d'utilisation que vous pouvez consulter en ligne.

https://apropos.erudit.org/fr/usagers/politique-dutilisation/ 


\title{
Pistes de solutions pour l'amélio- ration des relations entre les services techniques et les services au public
}

\author{
JOHANNE LAVOIE \\ Chef de section - Services techniques et système de gestion \\ Bibliothèque de Dollard-des-Ormeaux \\ jlavoie@ddo.qc.ca
}

Ce texte a été réalisé à l'EBSI, Université de Montréal, dans le cadre du cours BLT6123 Services techniques et automatisés, donné durant la session d'hiver 2005 par Benoit Ferland.

L EXISTE UN VIEUX MYTHE DE DIVERGENCE, voire de division profonde - le mythe des deux solitudes - entre les services techniques et les services au public dans les bibliothèques. Certes, le rôle de chaque service est différent: alors que le premier regroupe l'"ensemble des services d'acquisition, de traitement, de préparation matérielle, de préservation et de réparation des documents, ainsi que de création et de gestion des catalogues ${ }^{1}$ ", le second comprend $l^{\prime}$ " ensemble des services qui s'adressent directement aux usagers $^{2}$ », notamment la référence. Par contre, les deux services ont un objectif commun: répondre aux besoins des usagers. Pour que cet objectif soit atteint le mieux possible, il faut tout de même que services techniques et services au public travaillent main dans la main, ce qui n'est pas toujours le cas. Comment faire, alors, pour améliorer la communication et la coopération entre les deux services? Nous tentons, dans ce texte, de proposer des pistes de réponse à cette toujours épineuse question. Dans un premier temps, nous procédons à une analyse d'articles écrits sur le sujet, puis nous essayons de comprendre d'où provient cette division. Nous proposons finalement des pistes de solutions pour atténuer, voire éliminer ce problème. La solution qui nous semble, à priori, la plus facilement réalisable et la plus profitable à court terme serait d'affecter des bibliothécaires travaillant aux services techniques quelques heures par semaine aux services au public et vice-versa, afin que chacun prenne conscience des différentes activités se déroulant dans leur milieu de travail.

\section{Analyse des écrits sur le sujet}

À notre grande surprise, hormis un article de 2004 (Proctor) et un de 2000 (Folsom), la plupart des textes sur la question datent des années 1980 et du début des années 1990. En fait, le texte qui semble

\footnotetext{
1. Irène King, et al. Terminologie de base en sciences de l'information: volets 1 et 2. <http://www.ebsi.umontreal.ca/termino/index.htm> (consulté sur Internet le 3 mars 2005).

2. Ibid.
}

avoir lancé la réflexion sur la question est «On Doing Away with Technical Services Departments» de Michael Gorman, publié en 1979. Est-ce à dire que ce sujet n'est plus d'actualité? Peut-être. Pourtant, il existe toujours une difficulté de coopération certaine entre les services techniques et les services au public. Comme nous sommes encore majoritairement dans un modèle traditionnel des bibliothèques dans lequel services techniques et services au public sont séparés, les solutions proposées dans les textes des années 1980 sont, dans la majorité des cas, toujours envisageables aujourd'hui.

Plusieurs articles (Bing, Stevenson, Futas, Proctor) traitent de la division entre services techniques et services au public de manière générale. De nombreux textes soulignent que l'automatisation permet de repenser la structure traditionnelle des bibliothèques (Bing, Altmann, Davenport, Gorman, Futas). Deux textes, ceux de Margaret Bing (1999) et Sheila S. Intner (1987), portent sur l'image que les autres bibliothécaires ont des catalogueurs, image qui est souvent loin d'être reluisante. Enfin, plusieurs articles proposent des solutions pour rapprocher services techniques et services au public. La solution qui revient le plus souvent est l'assignation de catalogueurs aux services au public, plus particulièrement à la référence (Folsom, Leverence, Glascoff et Morrow, Eskov, Bierbaum). Un seul texte, celui de Sheila S. Intner (1989), propose l'idée inverse, soit qu'un bibliothécaire de référence ferait un bon catalogueur. Deux articles (Davenport, Hardin) présentent le cas d'un bibliothécaire qui partage son temps entre les deux services. Renee Beihl démontre l'importance de former les bibliothécaires aux activités des services techniques aussi bien quà celles des services au public. Ruby E. Miller et Barbara J. Ford (1990) expliquent les avantages de la création d'un comité dans lequel on retrouve des bibliothécaires œuvrant aussi bien aux services techniques qu'aux services au public. Enfin, Anna E. Altmann (1988) et, surtout, Michael Gorman (1983) proposent de repenser complètement la structure traditionnelle des bibliothèques. 


\section{D'où vient la division services techniques-services au public?}

"And it came to pass that when Kutta the Book God had made the first Library, she saw that it was good. She called the librarians together and divided them as a herder divides the sheep and the goats. To the first group she spoke, saying "You shall dwell in the light and serve the readers, and your glory shall be great». Then she turned to the second group and spoke, saying "You shall dwell in the darkness. Secret shall be your ways and hidden your practices. You shall not know the public, neither shall any reader know you. Go forth and classify ${ }^{3} . "$

Cette métaphore biblique de Michael Gorman illustre avec humour une situation qui est pourtant bien réelle. Bien sûr, elle n'explique pas les véritables raisons de la division qui existe entre services techniques et services au public. Selon Anna E. Altmann, deux raisons principales sont à la base de cette division. D'une part, les outils de travail nécessaires aux bibliothécaires des services techniques, alors sur papier, devaient être accessibles facilement: "The shelflist, the catalogue, and the order file take up a great deal of space and are not easily duplicated. Therefore, cataloguing and acquisition activities - technical services - are grouped and take place where the files are housed ${ }^{4}$.» D'autre part, la formation nécessaire pour devenir catalogueur ou bibliothécaire de référence est très longue. Cela signifie qu'il faudrait le double du temps nécessaire pour devenir à la fois efficace dans l'un et l'autre service.

Margaret Bing voit les choses un peu différemment. Selon elle, la division serait apparue après la Deuxième Guerre mondiale, alors qu'on assistait à une explosion de l'information et de la technologie, et à un changement dans la profession de bibliothécaire: "Unfortunately, with the rapid growth of large systems and networks, library service departments have become so large they seem to have taken a life of their own. During this process they lose track of other departments that make the library what it is, causing a dualism that does not really exist ${ }^{5}$.» Gordon Stevenson abonde dans le même sens, indiquant que chaque groupe de spécialistes fonctionne en vase clos, ayant très peu de communication l'un avec l'autre, ayant ses propres associations professionnelles, sa propre littérature professionnelle, ses propres valeurs, etc.

3. Michael Gorman. «On Doing Away with Technical Services». American Libraries, vol. 10, juillet-août 1979, p. 435 .

4. Anna E. Altmann. "The academic library of tomorrow: who will do what?». Canadian Library Journal, vol. $45, \mathrm{n}^{\circ} 3$, juin 1988, p. 148.

5. Margaret Bing. "The False Dualism: Technical Services vs. Public Services ». Journal of Library Administration, vol. 29, $\mathrm{n}^{\circ}$ 2, 1999, p. 24.
Certains réduiront cette division à ceci : d'un côté on retrouve les bibliothécaires qui s'occupent des livres, de l'autre les bibliothécaires qui s'occupent des gens. Malgré un fond de vérité, cette affirmation est beaucoup trop simpliste. En fait, tout bibliothécaire, qu'il soit aux services techniques ou aux services au public, travaille à répondre aux besoins des usagers de sa bibliothèque:

"The truth is that there is no division between reference and cataloging in terms of public service. You need both to keep your library functioning as a library. Actually there is no person in the library who is not doing public service. [...] it takes many people to deliver the final product, which is the acquiring, organizing, housing and delivery of information in all its varieties ${ }^{6} . »$

\section{Pistes de solutions}

Il importe de rétablir une communication efficace et une coopération réelle entre les services techniques et les services au public. Cela permettrait d'enrayer les tensions qui existent entre les deux groupes. En effet, les bibliothécaires de référence ont bien souvent une mauvaise image des catalogueurs et vice-versa, comme l'illustre Anna E. Altmann: «[...] cataloguers produce the perfect cataloguing record in total ignorance of the needs of the user, while reference librarians don't really understand their own catalogue ${ }^{7}$." De plus, de meilleures relations entre services techniques et services au public auraient probablement pour résultat d'augmenter la satisfaction des usagers.

Cinq pistes de solutions sont possibles pour diminuer et même éliminer, dans certains cas, ce clivage entre services techniques et services au public. Certaines solutions sont plus facilement réalisables que d'autres, qui demandent un changement plus profond des mentalités et des structures traditionnelles des bibliothèques. Qui plus est, une solution n'est pas nécessairement applicable à toutes les bibliothèques. Quoi qu'il en soit, ces pistes de solution peuvent tout de même servir à entamer une réflexion sur la question.

\section{Changer la dénomination « services techniques »}

Il est de notoriété publique que catalogueurs et bibliothécaires de référence ont une mauvaise image les uns des autres. Cependant, plus souvent qu'autrement, le bibliothécaire de référence est vu de façon plus positive, car c'est celui qui est «sur la ligne de feu» et qui répond directement aux besoins des

6. Ibid., p. 24

7. Anna E. Altmann. Op.cit., p.147. 
usagers. Son travail est donc plus visible et, partant, davantage valorisé. Quant aux catalogueurs, leur image est plus négative:

"For too long catalogers have been considered the poor relations in the library hierarchy. They are too often relegated to the basement or to the back room. [...] They receive none of the pats on the back given to the reference librarians, the "thank you» for help. Has anyone ever congratulated a cataloger on his or her choice of heading of fancy footwork with a 500 note 8 ?"

Pour obtenir une amélioration des rapports entre services techniques et services au public, il faut donc à tout prix changer l'image des catalogueurs. Une des façons d'y parvenir, selon Sheila $S$. Intner, est de trouver une autre dénomination aux services techniques. En effet, l'expression «services techniques» évoque un service de second plan, donc une connotation de subordination. Et subordination à qui? "Public service people. They select. We acquire. They plan. We catalog. They advise. We inventory. They program. We file. They serve the public. We serve the files, the materials, the shelf arrangement-all inanimate things ${ }^{9}$." Le terme technique, en lui-même, peut être mis en contraste avec le terme scientifique, ce qui contribue à appauvrir l'image associée aux bibliothécaires travaillant aux services techniques. Ces derniers sont vus comme des gens qui appliquent des règles. Pour Sheila S. Intner, «Nomenclature is a weapon». Souvent, on associe à l'importance d'une dénomination une certaine reconnaissance. Elle propose "Information Services" (services d'information), "Bibliographic Services" (services bibliographiques) ou encore «Bibliographic Information Services" (services d'information bibliographique). Elle retient ces termes, car ils mettent l'accent sur le fait que les services techniques fournissent de l'information. Et c'est souvent ce qui est oublié par les bibliothécaires mêmes qui œuvrent dans ce secteur: «Too often, I fear, they think of themselves as supporters of information services or as processors of information, but not as the purveyors of information services to anyone, not even the librarians whose services to the public they support. That is the greatest failure of the names we use $e^{10}$."

Sheila S. Intner suggère enfin que les services techniques soient plus visibles dans la bibliothèque, c'est-à-dire qu'ils ne soient pas relégués au dernier étage ou encore au sous-sol, là où ils risquent davantage d'être oubliés et mis de côté.

8. Gillian McCombs. «Public and Technical Services: the Hidden Dialectic». RQ vol. $28, n^{\circ} 2$, hiver 1988, p. 142 .

9. Sheila S. Intner. "The Technical Services Mystique». Technicalities, vol. 7 $\mathrm{n}^{\circ} 1$, janvier 1987, p. 8.

10. Ibid., p. 11.
Cette solution est intéressante, mais demande un changement de mentalité profond. Il n'est pas facile de se débarrasser d'une image préconçue que les gens entretiennent. C'est un long processus qui exige beaucoup de travail. Cependant, la modification de la dénomination pourrait être le déclencheur d'un tel processus.

\section{Créer un comité catalogage-référence}

Dans leur article, Ruby E. Miller et Barbara J. Ford (1990) évoquent l'exemple de la bibliothèque de la Trinity University, qui a mis en place un comité (Cataloging/Reference Committee) ayant pour but de rapprocher les services techniques et les services au public. Au départ, soit en 1979, le seul contact formel entre ces deux groupes consistait en une réunion mensuelle. Au tournant de 1982, le Cataloging/ Reference Committee a vu le jour, formé de catalogueurs et de bibliothécaires de référence. Il était alors plutôt perçu comme un forum de discussion et n'avait pas de cadre formel. D'ailleurs, il n'était pas obligatoire pour les bibliothécaires d'y participer. Cependant, étant donné l'intérêt des bibliothécaires (tous assistaient aux rencontres), il a pris de l'importance: une rencontre formelle était organisée à chaque mois, présidée à la fois par le directeur des services techniques et le directeur des services au public. Cette formule a contribué à améliorer les relations entre les deux groupes de bibliothécaires:

\section{"One of the benefits is that both catalogers and reference librarians can discuss problems in each area. Reference librarians do not always know the consequences for catalogers of making a change in the catalog. Catalogers do not always know how important a reques- ted change is for the reference librarians and the library users ${ }^{11}$."}

La formation d'un tel comité peut donc avoir des effets bénéfiques sur les relations entre bibliothécaires des services techniques et des services au public ainsi que sur les services aux usagers. Qui plus est, cette solution peut être implantée dans n'importe quelle bibliothèque, à condition qu'il y ait volonté de part et d'autre de participer à un tel comité.

\section{Offrir des séances de formation mutuelle}

La formation mutuelle peut être un bon moyen de rapprocher les bibliothécaires des services techniques et des services au public. En effet, la tension entre les deux groupes résulte bien souvent de l'incompréhension qu'ils ont l'un de l'autre. Dans cette optique de formation mutuelle, un catalogueur pourrait recevoir

11. Ruby E. Miller et Barbara J. Ford. "A Relationship Between Cataloging and Reference». Texas Library Journal, vol. 66, $\mathrm{n}^{\circ}$ 1, printemps 1990, p. 51. 
une formation sur l'aide à l'usager. Cela lui permettrait entre autres de prendre conscience de la manière dont les usagers utilisent le catalogue. Le bibliothécaire de référence, quant à lui, pourrait recevoir une formation sur le catalogue et être informé des derniers développements technologiques. Non seulement ces formations permettraient à chacun d'en savoir plus sur le travail de l'autre mais, en plus, les connaissances acquises dans ce contexte pourraient leur être utile dans leur travail respectif. Renee Beihl (1995) souligne les avantages de la formation mutuelle à la Fort Collins Public Library: «Training librarians and other staff in more than one library department can serve to narrow the divisions, build a stronger overall team, minimize the effects of temporary staff shortages, and improve

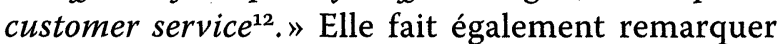
qu'à la suite de l'implantation de cette pratique, les bibliothécaires des services techniques ont démontré davantage de respect pour le travail de leurs collègues des services au public et vice-versa.

Cette solution nous apparaît fort intéressante. En effet, elle n'exige pas que chacun devienne polyvalent et puisse être compétent à la fois aux services techniques et aux services au public, mais bien que chacun ait conscience de toute la chaine documentaire dans sa propre institution. Cependant, tout comme dans le cas de la solution précédente, il doit y avoir un intérêt de la part des bibliothécaires à participer à des séances de formation mutuelle pour que ça fonctionne vraiment.

\section{Créer des postes combinant des activités liées aux services techniques et des activités liées aux services au public}

Cette solution est déjà appliquée dans plusieurs bibliothèques, mais à des degrés divers. En effet, dans certains cas, il s'agit d'un catalogueur qui fournit de l'aide à l'usager quelques heures par semaine; dans d'autres cas, beaucoup plus rares, des bibliothécaires partagent leur temps également entre les services techniques et les services au public.

\section{Le catalogueur au bureau de référence}

Il est plus fréquent de voir un catalogueur passer quelques heures aux services au public qu'un bibliothécaire de référence passer quelques heures aux services techniques. En effet, la littérature rapporte beaucoup de cas de catalogueurs mettant à profit leurs connaissances au bureau de référence. Bien souvent, il ne s'agit que de combler les heures où les bibliothécaires de référence sont absents du comptoir d'aide à l'usager, par exemple le soir et la fin de semaine. Le catalo-

12. Renee Beihl. Cross Training and Technical Services: Bridging the Gap. Colorado Libraries, vol. 21, $\mathrm{n}^{0} 3$, automne 1995, p. 52. Nous soulignons. gueur possède une expertise qui peut être intéressante pour les usagers: "Catalogers' thorough knowledge of subject headings and other access points can be extremely helpful in finding appropriate records ${ }^{13}$." Patricia A. Eskov a réalisé une enquête à ce sujet dans les années 1980. Elle s'est penchée sur l'assignation des catalogueurs à des tâches autres que le catalogage. Elle a relevé les aspects positifs d'une telle pratique:

«[...] heightened job interest among participants, better use of individual abilities, increased understanding between technical and public services, and more flexibility among library staff when coping with emergencies. Catalogers may become more attuned to patrons' needs by serving at the reference desk; reference librarians are better able to assist patrons in the use of the catalog if they have actual cataloging experience ${ }^{14}$. »

Sandy L. Folsom souligne, elle aussi, l'effet positif d'une telle pratique sur les relations entre services techniques et services au public: "In a way, it brings together the two «kinds» of librarians for the new dimension of library service ${ }^{15}$. " Par contre, assigner aux catalogueurs d'autres tâches que les leurs comporte aussi des inconvénients: " [...] fragmentation of time, lack of consistency in cataloging, problems with training, and difficulties involved in staff developing expertise in multiple areas $^{16}$. " De plus, comme cette façon de faire ne va que dans un sens (les bibliothécaires de référence ne sont pas appelés à participer aux tâches normalement attribuées aux bibliothécaires des services techniques), il n'est pas garanti d'éveiller la compréhension et le respect mutuel dans les deux services.

\section{Le bibliothécaire de référence au catalogage}

Les articles portant sur la situation inverse, c'est-àdire sur des bibliothécaires de référence affectés aux services techniques, sont plus rares. Pourtant, selon Sheila S. Intner, ceux-ci ont tout le potentiel pour devenir de bons catalogueurs: "[...] fewer reference librarians are hired with the understanding that they must put in a certain number of hours in the cataloging department, although this results in a great waste of sorely-needed expertise in the cataloging department for particular language or subject areas ${ }^{17}$.» Elle donne dans son article dix bonnes raisons pour lesquelles les

13. Sandy L. Folsom. "Out of the nest: the cataloger in a public services role". Library Collections, Acquisitions \& Technical Services, vol. 24, 2000, p. 67.

14. Patricia A. Eskov. "Catalog Librarians and Public Services - A Changing Role?». Library Resources and Technical Services, vol. 35, $\mathrm{n}^{\circ} 1$, janvier 1991, p. 83. Nous soulignons.

15. Sandy L. Folsom. Op.cit., p. 68. L'expression two «kinds» of librarians est de Michael Gorman.

16. Patricia A. Eskov. Op. cit., p. 83

17. Sheila S. Intner. "Ten Good Reasons Why Reference Librarians Would Make Good Catalogers». Technicalities, vol. 9, $\mathrm{n}^{\circ} 1$, janvier 1989, p. 14. 
bibliothécaires de référence feraient de bons catalogueurs. Il est certain que l'image négative que les bibliothécaires des services au public ont trop souvent des catalogueurs serait modifiée si l'on implantait dans une bibliothèque une telle façon de travailler. Par contre, le catalogage demande la compréhension de règles complexes, et le passage du catalogage vers le bureau de référence est sans doute plus aisé que le parcours inverse. Quoi qu'il en soit, il ne s'agit pas, ici non plus, d'un véritable partage des tâches, mais ce qui serait, selon nous, nécessaire pour améliorer les relations entre services techniques et services au public.

\section{The holistic librarian ou le bibliothécaire global}

La situation idéale serait la suivante: un bibliothécaire qui consacre la moitié de son temps à des tâches liées aux services techniques et l'autre moitié à des tâches liées aux services au public. En anglais, les expressions holistic librarian et holistic librarianship sont utilisées pour parler de cette réalité peu répandue. Le terme holistique, selon le Grand dictionnaire terminologique, «s'applique à toute démarche globalisante ou syncrétique où divers éléments, habituellement isolés, sont regroupés et coordonnés pour l'obtention plus efficace d'un résultat visé ${ }^{18}$ ». Nous n'avons pas trouvé dans la littérature d'équivalents français à ces expressions. Nous aurions pu traduire par «bibliothécaire holistique», mais nous préférons l'expression «bibliothécaire global».

La Penfield Library de la State University of New York College, à Oswago, a aboli la division services techniques / services au public au début des années 1970, en exigeant de tous ses bibliothécaires qu'ils travaillent au bureau de référence et qu'ils soient formés aux opérations effectuées aux services techniques. Le directeur de la bibliothèque souhaitait que les bibliothécaires deviennent des "généralistes », c'est-à-dire qu'ils soient compétents dans leur service, mais également fonctionnels dans les autres services de la bibliothèque. Une telle pratique a des effets positifs sur la division traditionnelle services techniques et services au public:

"As technical services staff were becoming involved in reference activities, reference staff were increasing their understanding of technical services operations. [...] differences between technical services and public services became even less distinct and the involvement of reference librarians in what had traditionally

18. Office québécois de la langue française. Grand dictionnaire terminologique. $<h t t p: / / w w w . o l f . g o u v . q c . c a /$ ressources/gdt_bdl2.html> (page consultée le 4 mars 2005). been technical services activities increased significantly ${ }^{19}$.»

Plus intéressant encore est le témoignage de Steve Hardin, qui travaille à la Cunningham Memorial Library de l'Indiana State University et qui est un véritable bibliothécaire global. En effet, il passe ses avant-midi aux services au public et ses après-midi aux services techniques. "Holistic librarianship is diverse, challenging, and, frankly, fun ${ }^{20}$ !" affirme-t-il. Bien sûr, un tel poste entraîne un certain nombre de problèmes, notamment le fait qu'il faut plus de temps au bibliothécaire pour devenir compétent dans les deux fonctions. De plus, il arrive souvent au bibliothécaire d'être interrompu dans son travail. Par exemple, Hardin raconte qu'un usager qu'il a aidé pendant son travail au bureau de référence peut revenir le voir alors qu'il est au catalogage. Cependant, il croit que les avantages sont plus importants que les désavantages:

"The variety of the work keeps things from getting stale. And it's a great satisfaction to see how the things you do in Tech Services are used by both the librarians in Public Services and by the patrons themselves. [...] And it doesn't hurt to have someone reasonably familiar with how the actions of one department affect the operations of another ${ }^{21}$."

Plus près de nous, la bibliothèque de l'Université Laval à Québec fonctionne un peu de cette façon. En effet, les bibliothécaires sont appelés à effectuer un certain nombre de tâches liées aux disciplines qui leur sont assignées: développement des collections, référence spécialisée, préparation d'outils de recherche documentaires, mais également indexation et classification des ressources de leur discipline.

Il en va de même à la bibliothèque de Boisbriand. En effet, les deux techniciennes partagent leur temps également entre les services techniques et les services au public. Comme le souligne Ghislaine Lauzon, directrice de la bibliothèque: "œuvrer à la fois aux services techniques et au public signifie détenir des priorités de travail claires et justes; ainsi, c'est aux besoins des clients que la coordonnatrice des services techniques se réfère pour sélectionner les documents à traiter en tout premier lieu ${ }^{22} . »$

Avec le bibliothécaire global, la distinction entre services techniques et services au public est appelée à s'estomper. Généralement, ce ne sont pas tous les bibliothécaires d'une institution qui occupent ce

19. Sara Evans Davenport. «The Blurring of Divisional Lines Between Technical and Public Services: An Emphasis on Access». The Reference Librarian, $\mathrm{n}^{0} 34,1991$, p. 49.

20. Steve Hardin. "The Servant of Two Masters». Technicalities, vol. $13, \mathrm{n}^{\mathrm{o}} 7$, juillet 1993, p. 11.

21. Ibid., p.12

22. Ghislaine Lauzon. "Organisation de la chaîne documentaire dans une petite bibliothèque publique: le cas de Boisbriand». Argus, vol. 21, n ${ }^{\circ}$, automne 1992, p. 9. Merci à $\mathrm{M}^{\mathrm{me}}$ Lauzon pour les explications qu'elle m'a fournies par téléphone. 
genre de poste, mais seulement certains d'entre eux. Ces personnes servent donc de lien entre les services techniques et les services au public.

La «bibliothéconomie holistique» règle en partie le problème de la division entre services techniques et services au public. Par contre, une telle solution n'est pas facile à mettre en place, surtout dans une grande bibliothèque. Les deux autres solutions présentées ici, soit d'affecter un catalogueur au bureau de référence et de confier à un bibliothécaire de référence du travail attribué généralement à un bibliothécaire des services techniques, sont sans doute un peu plus facilement applicables et permettent tout de même de rapprocher les deux services. Ce serait déjà un pas dans la bonne direction.

\section{Repenser la structure traditionnelle des bibliothèques: le modèle de Gorman}

L'une des raisons qui ont expliqué l'apparition de la division entre services techniques et services au public était le regroupement des catalogueurs autour des documents de travail qui leur sont propres et qui étaient alors en format imprimé. Cependant, avec l'avènement de l'automatisation, il peut maintenant en être autrement: «[...] the metamorphosis of the immovable paper files into online files removes the physical impediment to the decentralization of cataloguing: once the files are on-line, and in effect located wherever there is a terminal, each subject division of the library can carry out the full range of library

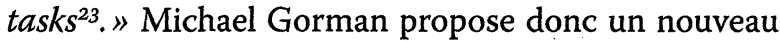
modèle pour les bibliothèques, modèle qui ferait en sorte que la division services techniques et services au public disparaîtrait complètement. Voici ce qu'il suggère:

"[...] to create structures in which groups of librarians are defined not by which aspect of professional librarianship they practice (cataloguing, reference work, etc.) but by the area of service in which they exercise their skills across the whole range of professional librarianship. That is, instead of being limited by the use of one skill or one group of skills, the librarian in this model will be limited only by the type of library user which they serve. In a small library that type might include all users of the library and, in some senses, the model already operates; in a larger library, the type of library user might be defined by subject (e.g., Humanities, Law), by age or educational level (e.g., children, undergraduates), by area (e.g., Latin American studies), or by any other cha-

23. Anna E. Altmann. Op.cit., p. 149. racteristic of division which is appropriate to the mission of the library ${ }^{24}$."

À cette réorganisation s'ajouterait une séparation entre le travail qui doit être fait par un professionnel et le travail qui peut être fait par un non-professionnel. Si l'on prend l'exemple du catalogage: le catalogage original serait fait par le bibliothécaire, alors que le catalogage dérivé serait pris en charge par un nonprofessionnel. Les bibliothécaires se verraient donc attribuer toutes les tâches dites professionnelles: développement de collections, catalogage original, référence, formation des utilisateurs, etc. Une telle réorganisation du travail apporterait selon Michael Gorman de nombreux avantages: "The re-grouping of professional librarians around subject and other user categories will not only bring better services to the library users they serve, it will also produce more job satisfaction and more career potential for the librarians involved ${ }^{25}$.»

Le modèle de la bibliothèque de l'Université du Québec à Chicoutimi (UQAC), en place depuis 1982, diffère du modèle traditionnel et constitue en cela un bon exemple de ce que propose Michael Gorman. D'un côté, on retrouve la division des services d'information documentaire, qui se subdivise en «zones». Les zones correspondent à des secteurs disciplinaires, où techniciens et bibliothécaires effectuent un certain nombre de tâches liées à une discipline, par exemple la référence, le catalogage, l'indexation et la classification. De l'autre côté, on retrouve la division des services généraux, où sont regroupées toutes les activités qui ne concernent pas les secteurs disciplinaires, comme les acquisitions, le prêt et le rayonnage. Ce modèle fait en sorte, comme l'explique Gilles Caron, directeur de la bibliothèque de l'UQAC:

" [qu'] il n'y a plus de tiraillements entre les deux divisions, tiraillements qui sont omniprésents dans le modèle traditionnel où chaque grande division (services techniques et services au public) possède sa culture propre et sa vision souvent différente, sinon conflictuelle, du service à offrir au client. À l'UQAC, les personnels des deux (2) divisions vivent ce contact permanent avec le client, ce qui a pour effet de modifier radicalement la perspective ${ }^{26}$.»

La solution proposée par Michael Gorman est sans aucun doute la meilleure, car elle permet d'éliminer complètement la division entre services techniques et services au public. Par contre, la mettre en application

\footnotetext{
24. Michael Gorman. "The Ecumenical Library». The Reference Librarian, $\mathrm{n}^{\circ} \mathrm{9}$, automne-hiver 1983, p. 57.

25. Ibid., p. 63.

26. Gilles Caron. Douze ans plus tard: Le modèle organisationnel en opération à la bibliothèque de l'Université du Québec à Chicoutimi, 1994, p.13. <http://sdeir. uqac.ca/doc_numerique/format/PDF/13827342.pdf> (page consultée le 8 mars 2006). Merci à M. Caron pour les explications qu'il ma fournies par téléphone.
} 
ne va pas de soi, plus particulièrement dans de grandes bibliothèques. Cela demande toute une réorganisation du travail. Qui plus est, un tel changement ne plairait peut-être pas aux bibliothécaires, car leur travail serait bouleversé. On dit souvent que les services techniques et les services au public sont deux mondes. Le bibliothécaire qui aime faire du catalogage n'appréciera peut-être pas devoir faire de la référence et vice-versa. Le modèle de Michael Gorman est donc un modèle fort intéressant, mais qui ne risque pas de faire l'unanimité.

\section{Conclusion}

La division qui existe entre services techniques et services au public est ancienne. Son apparition est liée, entre autre, à des raisons d'ordre pratique. Pour améliorer le fonctionnement de nos bibliothèques, il importe cependant de rapprocher les deux services. Comme le fait remarquer Elizabeth Futas: "An understanding of what the other does, and deciding how to help each other achieve common goals and objectives, is essential ${ }^{27}$." Nous avons présenté quelques pistes de solutions pour atténuer ou même éliminer la division entre services techniques et services au public: changer la dénomination «services techniques», créer un comité catalogage-référence, faire de la formation mutuelle, créer des postes de "bibliothécaire global» et restructurer les services selon le modèle de Michael Gorman. Nous avions, en introduction, émis l'hypothèse que la solution la plus réalisable et la plus intéressante à court terme serait d'affecter, quelques heures par semaine, un catalogueur au comptoir d'aide à l'usager et un bibliothécaire de référence au catalogage, afin qu'ils prennent connaissance du travail des autres. Cette solution est en effet intéressante, mais il n'existe pas vraiment de solution miracle, applicable à tous les milieux. C'est à chaque institution de choisir celle qui lui convient le mieux. Il est certain que la division services techniques et services au public ne pourra pas être abolie du jour au lendemain. Ce qui serait tout de même souhaitable à court terme, c'est de voir apparaître non seulement une volonté d'améliorer les relations entre les deux groupes, mais également d'accompagner cette volonté de gestes concrets. $\odot$

\section{Sources consultées}

Altmann, Anna E. 1988. The Academic Library of Tomorrow: Who Will Do What? Canadian Library Journal, vol. $45, \mathrm{n}^{\circ} 3$, p. 147152.

Beihl, Renee. 1995. Cross Training and Technical Services: Bridging the Gap. Colorado Libraries, vol. 21, $\mathrm{n}^{\circ}$ 3, p. 52-53.
Bierbaum, Esther Green. 1993. The Convenience of the Reader: How Technical Services Can Contribute to Effective Adult Services. $R Q$, vol. 32, $\mathrm{n}^{\circ} 4$, p. 464-467.

Bing, Margaret. 1999. The False Dualism: Technical Services vs. Public Services. Journal of Library Administration, vol. 29, $\mathrm{n}^{\circ}$ 2, p. 23-28.

Caron, Gilles. 1994. Douze ans plus tard: Le modèle organisationnel en opération à la bibliothèque de l'Université du Québec à Chicoutimi, $32 \mathrm{p}$.<http://sdeir.uqac.ca/doc_numerique/ format/PDF/13827342.pdf> (page consultée le 8 mars 2006).

Davenport, Sara Evans. 1991. The Blurring of Divisional Lines Between Technical and Public Services: An Emphasis on Access. The Reference Librarian, $\mathrm{n}^{\circ}$ 34, p. 47-53.

Eskov, Patricia A. 1991. Catalog Librarians and Public Services - A Changing Role? Library Resources and Technical Services, vol. $35, \mathrm{n}^{\circ}$ 1, p. 76-86.

Folsom, Sandy L. 20oo. Out of the Nest: The Cataloger in a Public Services Role. Library Collections, Acquisitions \& Technical Services, vol. 24, p.65-71.

Glascoff, Ann et Lydia Morrow. 1990. Training Technical Services Staff to Provide Public Service. Illinois Libraries, vol. $72, \mathrm{n}^{\circ} 8$, p. 611-613.

Gorman, Michael. 1979. On Doing Away with Technical Services Departments. American Libraries, vol. 1o, p. 435-437. 1983. The Ecumenical Library. The Reference Librarian, $\mathrm{n}^{\circ} 9$, p. 55-64.

Hardin, Steve. 1993. The Servant of Two Masters: An Account of One Librarian's Adventures in Holistic Librarianship. Technicalities, vol. 13, $\mathrm{n}^{\circ} 7$, p.11-12.

Intner, Sheila S. 1987. The Technical Services Mystique. Technicalities, vol. 7, $\mathrm{n}^{\circ} 1$, p. 8-11. 1989. Ten Good Reasons Why Reference Librarians Would Make Good Catalogers. Technicalities, vol. 9, $\mathrm{n}^{\circ} 1$, p.1416.

King, Irène et al. Terminologie de base en sciences de l'information: volets 1 et 2. <http://www.ebsi.umontreal.ca/termino/index. htm > (page consultée le 3 mars 2005).

Lauzon, Ghislaine. 1992. Organisation de la chaîne documentaire dans une petite bibliothèque publique: le cas de Boisbriand. Argus, vol. 21, no 2, p. 8-14.

Leverence, Mari Ellen Quirk. 1996. Utilizing the Technical Services Staff on a Public Service Desk. Technical Services Quarterly, vol. 13, $\mathrm{n}^{\circ}$ 2, p.1-13.

McCombs, Gillian. 1988. Public and Technical Services: the Hidden Dialectic. $R Q$, vol. 28, $\mathrm{n}^{\circ}$ 2, p.141-145.

Miller, Ruby E. et Barbara J. Ford. 1990. A Relationship Between Cataloging and Reference. Texas Library Journal, vol. $66, \mathrm{n}^{\circ} 1$, p. 48-51.

Office québécois de la langue française: Grand dictionnaire terminologique. <http://www.olf.gouv.qc.ca/ressources/gdt_bdl2.html> (page consultée le 4 mars 2005).

Proctor, Edward. 2004. Mind the Gap.American Libraries, vol. 35 , $\mathrm{n}^{\circ} 5, \mathrm{p} .46-48$.

Salony, Mary F. A 1998. Selected Bibliography of Recent Publications on the Relationship Between Public Services and Technical Services. Southeastern Librarian, vol. 47, $\mathrm{n}^{\circ} 4$, p. 30-33.

Stevenson, Gordon. 1983. The Nature of the Problem, If It Is a Problem. The Reference Librarian, $\mathrm{n}^{\circ}$ 9, p. 3-7.

27. Citée par Gillian McCombs. Op. cit., p.141. 